\title{
C.R. Goldfarb, M. Chamarthy, F. Ongseng, S.R. Parmett, D.L. Bushnell, R. Mirtcheva-Trocheva, U. Sen, L.S. Zuckier. Nuclear Medicine Board Review: Questions and answers for self-assessment, 4th Edition
}

Thieme Medical Publishers, 2018. ISBN 978-1-62623-471-0

Luigi Mansi ${ }^{1}$

Published online: 10 September 2018

(C) Springer-Verlag GmbH Germany, part of Springer Nature 2018

This is a fundamental book for all seeking to prepare themselves well for the Nuclear Medicine Board Examination. The success of the editorial approach is clearly evidenced by this fourth edition, appearing almost 20 years after the first one in 1998. Since then nuclear medicine has almost completely changed, with the spread of imaging based mainly on PET/ $\mathrm{CT}$, the appearance of PET/MRI, and the consolidation of SPECT/CT as a major tool in traditional nuclear medicine using gamma emitters. Furthermore, new radiopharmaceuticals have been introduced into clinical practice and there have been incredible developments in radionuclide therapy, that now also uses alpha emitters. In this context, new terms, philosophies and indications have appeared with the main references to molecular imaging, theranostics and tailored medicine. In this scenario, procedures utilizing radiopharmaceuticals, in both diagnosis and therapy, have reached a primary role not only in research, but also in routine practice.

The book is authored by a group of experts including C. Richard Goldfarb, Murthy R. Chamarthy, Fukiat Ongseng, Steven R. Parmett, David L. Bushnell Jr., Rosna MirtchevaTrocheva, Urmi Sen and Lionel S. Zuckier, who work in different institutions, but are united by significant didactic experience, noticeably expressed in all the fields involved in the examination.

As is already clear from the title, the text is mainly structured on the basis of an interactive question-and-answer format, that is particularly helpful for fast assimilation of relevant concepts that must remain well impressed. It is evident that this publication cannot be used alone, being only the accompanying final or initial support for consolidating information that has to be acquired from more extensive and specific volumes. The book, comprising 373 pages, is structured into 24 chapters organized in four parts: (1) Essentials of Nuclear Medicine; (2) Single Photon Applications; (3) Positron Emission Tomography/Computed Tomography; and (4) Radionuclide Therapy. In total, 2,250 questions are included that cover both consolidated and old issues and newer ones, accompanied by almost 200 high-quality images. The question formats include multiple choice, matching test, and true/false. Three appendixes (how to recognize the wholebody scans; must-know calculations, scintigrams and concepts; and tips on examination preparation) are also included.

As is clear, the fundamental target of this book includes all who are interested in acquiring certification (or recertification) by the American Board of Radiology, the American Board of Nuclear Medicine, the Certification Board of Nuclear Cardiology, and the Nuclear Medicine Technology Certification Board. Nevertheless, also considering its low cost, the book may be suggested to all residents and students worldwide and to professionals who want to consolidate or refresh their knowledge in the field.
Luigi Mansi

mansi.luigi@libero.it

1 Interuniversity Research Center for Sustainability (CIRPS), Naples, Italy 